\title{
Analysis of Changes in Physical Strength over Time in Recent School-Age Students: Proposal for Multi-year Span Evaluation Chart with the O Model
}

\author{
Ken-ichi Watanabe ${ }^{1, ~}$, Katsunori Fujii ${ }^{2}$, Keiko Abe ${ }^{1}$, Yuki Kani ${ }^{1}$, Kan-ichi Mimura ${ }^{1}$ \\ ${ }^{1}$ Faculty of Education, Osaka Seikei University, Osaka-City, Japan \\ ${ }^{2}$ Graduate School of Business Administration and Computer Science, Aichi Institute of Technology, Toyota-City, Japan \\ Email address: \\ watanabe-k@osaka-seikei.ac.jp (Ken-ichi W.), fujii@aitech.ac.jp (Kastunori F.), abe-ke@osaka-seikei.ac.jp (Keiko A.), \\ kani@osaka-seikei.ac.jp (Yuki K.), mimura@osaka-seikei.ac.jp (Kan-ichi M.) \\ ${ }^{*}$ Corresponding author
}

\section{To cite this article:}

Ken-ichi Watanabe, Katsunori Fujii, Keiko Abe, Yuki Kani, Kan-ichi Mimura. Analysis of Changes in Physical Strength over Time in Recent School-Age Students: Proposal for Multi-year Span Evaluation Chart with the O Model. American Journal of Sports Science.

Vol. 9, No. 1, 2021, pp. 17-26. doi: 10.11648/j.ajss.20210901.13

Received: February 17, 2021; Accepted: March 2, 2021; Published: March 10, 2021

\begin{abstract}
For school-age children to lead healthy lives, they need suitable physical abilities for those lives. Together with this, measurements of physical strength and motor ability to assess those abilities could be considered essential. The physical strength of children has declined in recent years, and City $\mathrm{O}$ in the Kansai area of Japan has implemented an action plan to address the problem. This action plan was developed from a survey continued over seven years of the physique, physical strength, and motor abilities of fifth grade elementary school students and second year junior high school (seventh grade) students. To assess the efficacy of City O's action plan, this study analyzed trends in strength and motor ability over seven years to examine whether trends were rising or falling. The method adopted for this plan was to measure the same items as those in the strength and motor ability survey conducted by the Ministry of Education, Culture, Sports, Science and Technology. The wavelet interpolation model was applied to the multi-year trends in the results obtained over seven years to examine whether they were improving or declining. An improving trend was seen with time in the physical strength and motor ability of second-year junior high school girls. One may infer that behind the improving trend over time were the effects from City O's action plan. The background for the improving trend seen over the years seen in second year junior high school girls but not boys may include that girls were originally less active than boys and but were encouraged to be active by the action plan. Because the second year of junior high school is the period of late puberty for girls, and is a time when they are approaching their developmental peak in physical strength, it may be inferred that the increase in physical strength was affected by factors in the action plan. In this study, a new evaluation chart that considers trends over years was established using the wavelet interpolation model, and the efficacy of the multi-year span evaluation chart for physical strength is presented as the $\mathrm{O}$ model.
\end{abstract}

Keywords: Multi-year Span Evaluation, Physical Ability, Secular Change Curve

\section{Introduction}

The problem of decreased physical strength in youth goes back to the finding of decreased physical strength in a conscription survey of American soldiers sent to the Korean civil war between the north and south that broke out on the peninsula in 1950 . However, it is unlikely that the problem simply spread from there to become the problem of decreased physical strength in the youth of Japan. In a life-or-death battle, inferior physical strength can be fatal and the need to increase physical strength is obvious. Unlike this, the tendency for decreased physical strength in recent years can be said to be a result of the transition to a society of convenience produced by high economic growth in Japan. It has also been pointed out that the sleeping time of junior high school and high school students has decreased as a result of the more intense competition in high school and university entrance 
examinations, which is another factor in the decrease physical strength. While there are unlikely to be improvements unless some kind of measures are taken to counter decreased physical strength, can we really hope for meaningful improvements in physical strength with the adoption of sustained exercise habits? Nakai and Demura [1] reported from longitudinal measurements and surveys over three years in male technical school students that continuous exercise increased muscle power, whole-body endurance, and flexibility. Although that report is somewhat dated, reports by Shimada et al. [2, 3] also showed improved agility, muscle endurance, and whole-body endurance in groups who performed more continuous exercise. Of course, they stated that groups which exercised with lower frequencies had inferior whole-body endurance, muscle endurance, and agility. They also reported that regardless of age, continuous exercise basically had positive effects on preserving and enhancing physical strength and health, even in elderly people [4]. Therefore, a picture could also be drawn in which further continuous exercise increases physical strength and motor ability in the school years.

The information needed to maintain and improve physical strength requires an understanding of current levels of physical strength. Fortunately, in Japan new physical fitness measurements by the Ministry of Education, Culture, Sports, Science and Technology were officially started in 1999, following a trial in 1998. Physical strength and motor ability surveys conducted by the Ministry of Education, Culture, Sports, Science and Technology are currently reported every year, but as is well known these surveys started at the time of the Tokyo Olympics in 1964. They are based on the Sports Promotion Law established in 1961, and are conducted according to the Sports Test reported by the Health and Physical Education Council (1963). The aim of this sports test was clearly to develop sports and raise the physical strength of Japanese citizens.

These statistical surveys comprise a very valuable system that is unparalleled in the world, and Nishijima $[5,6]$ investigated the decline in physical strength in children and adolescents based on them. He showed a decline in physical strength from the peak in 1980 to 1985, which continues even today. Hatta [4], Sasaki [7], and Matsumoto [8] investigated annual trends in physical strength of university students, and while the trends stagnated in some items, they indicated an overall decline since 1985.

Evaluations of physical strength and motor ability by the Ministry of Education, Culture, Sports, Science and Technology in recent years show that the decline has bottomed out, but we can also see that it has not caught up with the previous level of 1980-1985. However, is it right to seek the past high standard of physical fitness in today's youth? It may be that high levels of physical strength are not that desirable in the environment of today's young people. Fujii et al. [9] introduced the concept of optimum valid physical fitness, the idea that when thinking about the level of physical strength suited to an adequate physique, having the physical strength appropriate to one's lifestyle and behavior is the best element in bringing people closer to health. With regard to the physical strength required by young people today, Hayakawa et al. [10] said that optimal valid health and physical strength is determined not by having the physical strength of a soldier or athlete but by having physical strength suited to their goals.

In this study, we analyzed the changes over time in schoolchildren in recent years from a longitudinal survey of physique, physical strength, and motor ability over seven years in children in the fifth grade of elementary school and the second year of junior high school (seventh grade), conducted by the Ministry of Education, Culture, Sports, Science and Technology. This was used to examine the effects of the action plan of City $\mathrm{O}$ in the Kansai area of Japan that was implemented to counter declining physical strength. Different from the analysis results reported each year by the Ministry of Education, Culture, Sports, Science and Technology, we examined whether there was a rising or falling trend by applying the wavelet interpolation model to the changes over seven years. However, there is no established technique to evaluate the process over time in rising and falling trends. The Ministry of Education, Culture, Sports, Science and Technology also has not proposed such a method. Therefore, in this study we established a new evaluation chart that considered changes over time using the wavelet interpolation model, and we propose a multi-year span evaluation chart for physical strength as the O Model.

\section{Methods}

\subsection{Subjects}

The subject data were measurements of physique, physical strength, and motor ability from the 2013 to 2019 school years for boys and girls in the fifth grade of elementary school and the second year of junior high school in City $\mathrm{O}$ in the Kansai area. In addition, data for boys and girls in the fifth grade of elementary school and the second year of junior high school from the results of physical strength and motor ability surveys published by the Ministry of Education, Culture, Sports, Science and Technology for that same period were used. The measured items included physique items (height, weight, BMI), and physical strength items (grip strength, repeated side step, sit and reach, sit-up, endurance running, 50-m dash, standing long jump, 20-m shuttle run, and softball throw).

\subsection{Analysis}

The mean value and standard deviation of physical strength test results for boys and girls in the fifth grade of elementary school and second year of junior high school were calculated for the 2013 to 2019 school years. A one-way analysis of variance was performed for physique and physical strength items between annual trends, and statistical differences in the fluctuations in trends over time were analyzed. The wavelet interpolation model was applied to the mean values for which statistical differences were seen in school years and physical strength items. Figure 1 shows of graph of the wavelet interpolation model applied to the trends over time from 2013 to 2019 in sit-up by girls in the second year of junior high school. A rising trend over the years is clearly shown. This kind of graph was made for all physical strength items in boys and girls in the fifth year of elementary 
school and second year of junior high school.

The wavelet interpolation model was also applied to the mean values $\pm 0.5 \mathrm{SD}$ and the mean values $\pm 1.5 \mathrm{SD}$. Figures 2-4 are the trends over time with the wavelet interpolation model applied to repeated side step, sit-up, and 20-m shuttle run, and are also evaluation charts for multi-year spans. As is clear when also looking at the trends over the years in the mean values for these three items, they are evaluation charts showing rising trends with time. These time span evaluation charts were also constructed for the other items.

\subsection{Wavelet Interpolation Model (WIM)}

The wavelet interpolation model (WIM) is a method in which a growth distance curve is drawn with interpolation between data with a wavelet function (Meyer's mother wavelet is the base function), the distance curve drawn is differentiated and the obtained growth velocity curve is derived. The growth distance values at the pubertal peak and the age of menarche were investigated in this study to approximately describe the true growth curve from the given growth data. The characteristics of the wavelet interpolation model are that local phenomena are read sensitively and the approximation accuracy is very high. The details of the theoretical background and the grounds for its effectiveness have already been described in Fujii's previous research [11-13], so the data analysis algorithm using the wavelet interpolation model is omitted here. In this study, especially, the wavelet interpolation model was applied to the age distance value of annual trend of physical fitness from 2013 to 2019 years in second year girls' junior high school (seventh grade) students.

\section{Results}

\subsection{Analysis of Trends over Time in Physique and Physical Strength with the Wavelet Interpolation Model}

Physique, physical strength, and motor ability were measured over the years from 2013 to 2019 in boys and girls in the fifth grade of elementary school and the second year of junior high school. Table 1 gives the statistical values showing the trends over time in second year junior high school girls in whom changes were seen over time. No significant differences were seen in the results of one-way analysis of variance for multi-year trends in physique and physical strength for other school years: fifth grade boys, second year junior high school boys, and fifth grade girls. However, a significant difference was seen in physical strength for second year junior high school girls $(\mathrm{P}<0.05)$. Therefore, the wavelet interpolation model was applied to the trends over the years of physical strength items (grip strength, repeated side step, sit and reach, endurance running, sit-up, 50-m dash, standing long jump, 20-m shuttle run, and softball throw) for second year junior high school girls.

The results of applying the wavelet interpolation method are shown in Figures 1 to 6 . These figures are for items that showed particularly large trends over years. First, although graphs are not shown, grip strength declined temporarily in 2015 but increased again in 2017 and showed fluctuations over the years. It cannot be said to have increased. A gradually increasing trend is seen in sit-ups, and an increasing trend was seen over the years (Figure 1). Sit-and-reach, repeated side step, 50-m dash, and 20-m shuttle run also showed increasing trends over the years (Figures 2 to 5). The standing long jump showed an increasing trend until 2016, a temporary decrease in 2017, and then an increase again until 2019. As a multi-year trend it showed an increase (Figure 6). The softball throw showed a fluctuating pattern, with a temporary decrease in 2014, an increase in 2017, and another decrease in 2019. Finally, endurance running showed temporarily faster times in 2014, and then gradually slower times until 2019. The increasing trend over the years in physical strength in second year junior high school girls in City $\mathrm{O}$ is notable.

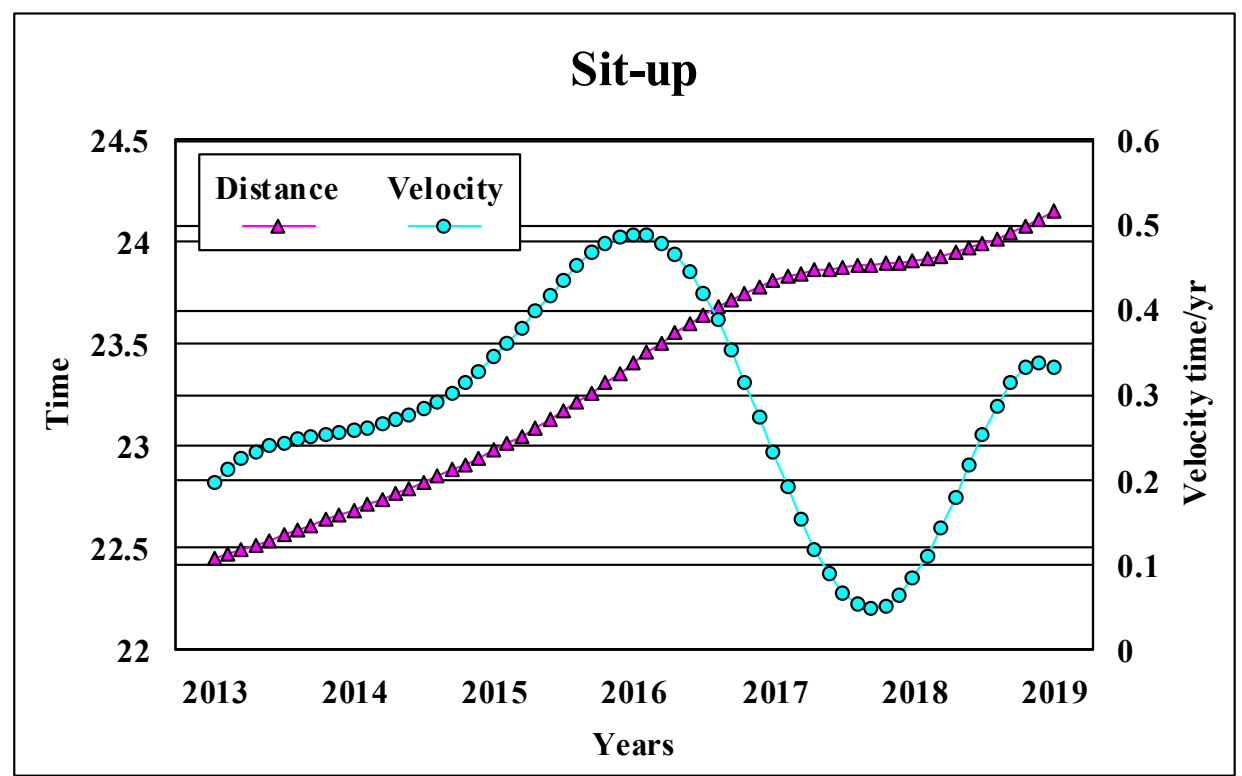

Figure 1. The secular change curve of sit-up by wavelet interpolation model (The second grade at a junior high school girls). 


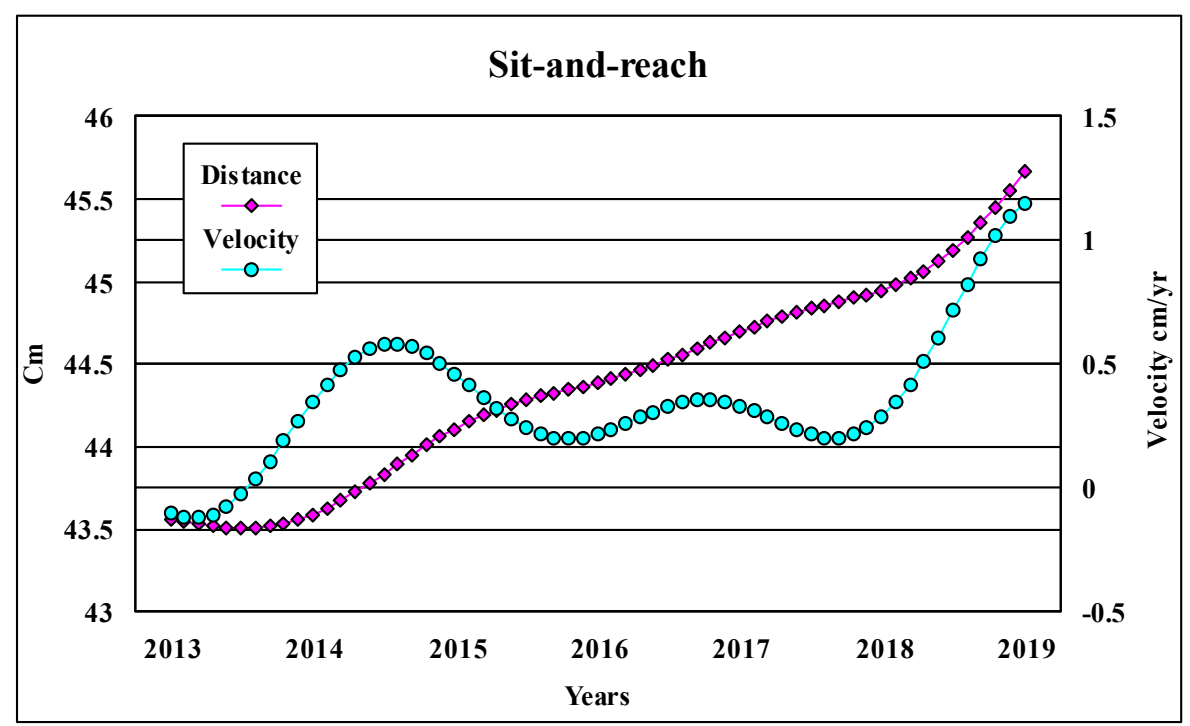

Figure 2. The secular change curve of sit-and-reach by wavelet interpolation model (The second grade at a junior high school girls).

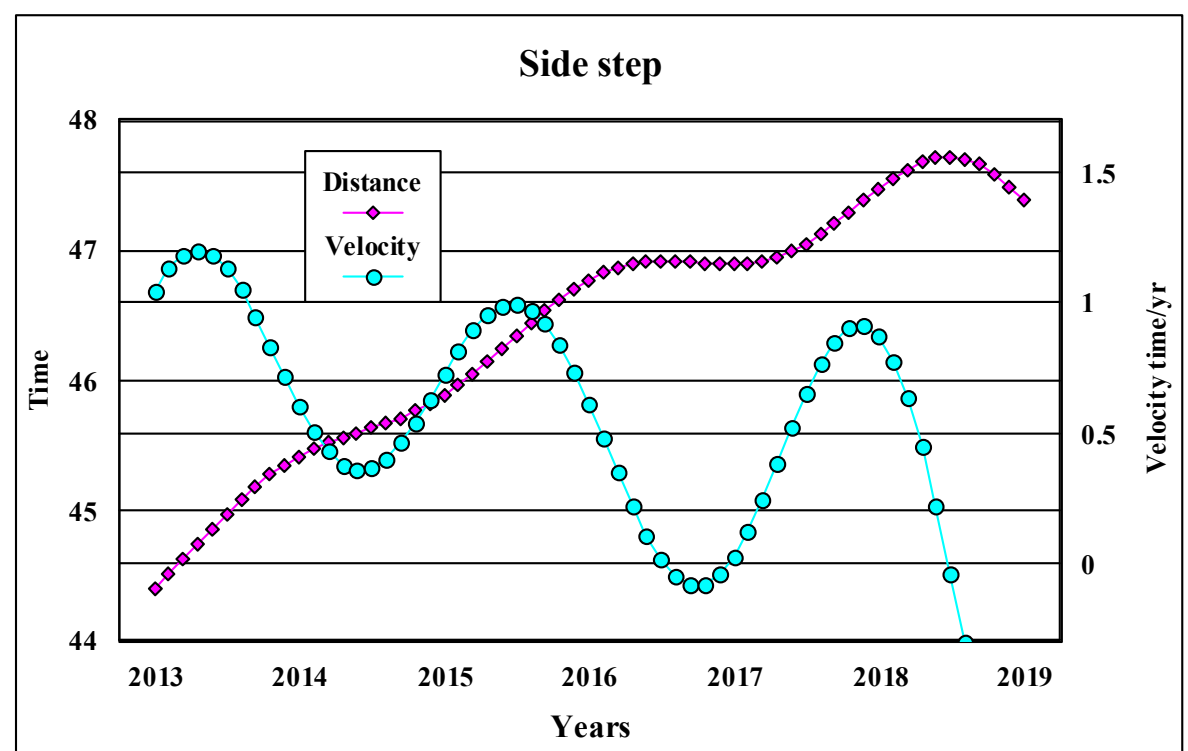

Figure 3. The secular change curve of side step by wavelet interpolation model (The second grade at a junior high school girls).

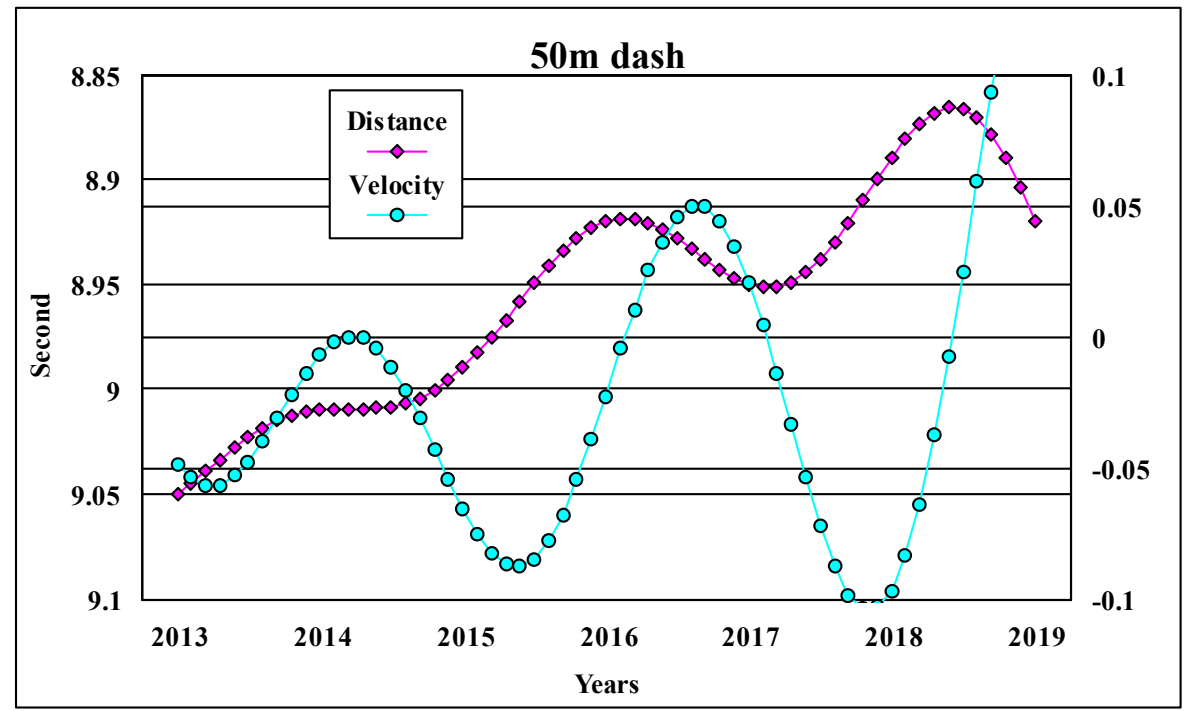

Figure 4. The secular change curve of $50 \mathrm{~m}$ dash by wavelet interpolation model (The second grade at a junior high school girls). 


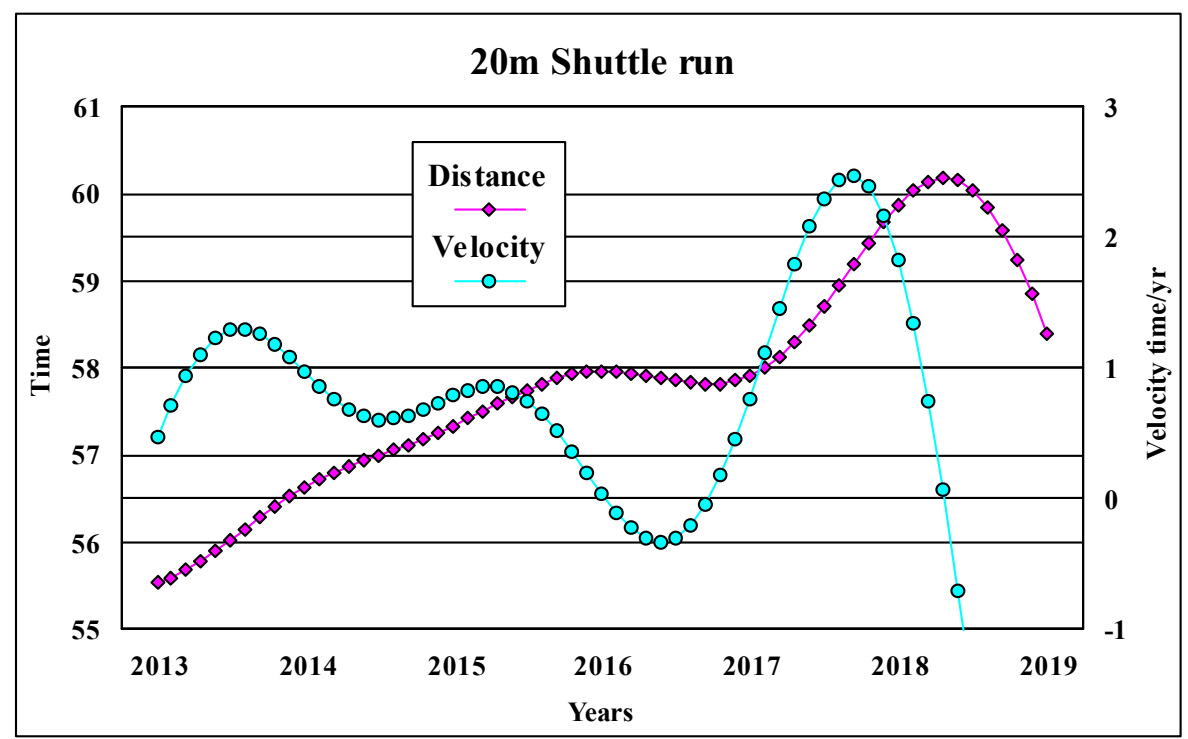

Figure 5. The secular change curve of $20 \mathrm{~m}$ shuttle run by wavelet interpolation model (The second grade at a junior high school girls).

Table 1. Annual statistics of physique and physical fitness in the second grade at a junior high school girls from 2013 to 2019 years

\begin{tabular}{|c|c|c|c|c|c|c|c|c|c|c|c|c|c|}
\hline year & & Height & Weight & $\begin{array}{l}\text { Grip } \\
\text { strength }\end{array}$ & Sit-up & $\begin{array}{l}\text { Sit-and } \\
\text { reach }\end{array}$ & $\begin{array}{l}\text { Side } \\
\text { step }\end{array}$ & $\begin{array}{c}\text { Endurance } \\
\text { running } \\
\end{array}$ & $\begin{array}{l}20 \mathrm{~m} \\
\text { shuttle run }\end{array}$ & $\begin{array}{l}\mathbf{5 0 m} \\
\text { dash }\end{array}$ & $\begin{array}{l}\text { Standing } \\
\text { long jump }\end{array}$ & $\begin{array}{l}\text { Hand-ball } \\
\text { throwing }\end{array}$ & $\begin{array}{l}\text { Total physical } \\
\text { fitness score } \\
\end{array}$ \\
\hline \multirow{4}{*}{2013} & $\mathrm{n}$ & 7707 & 7307 & 7995 & 7827 & 7960 & 7838 & 1915 & 6198 & 7742 & 7848 & 7828 & 6968 \\
\hline & mean & 155.07 & 46.55 & 24.16 & 22.45 & 43.56 & 44.4 & 307.17 & 55.54 & 9.05 & 163.23 & 13.07 & 47.03 \\
\hline & $\mathrm{SD}$ & 5.39 & 7.33 & 4.69 & 5.62 & 9.22 & 6.66 & 45.02 & 20.33 & 0.82 & 23.13 & 4.11 & 10.69 \\
\hline & $\begin{array}{l}\text { T-score } \\
\text { (National comparison) }\end{array}$ & 50.5 & 49.7 & 50.9 & 49 & 48.4 & 48.7 & 46.8 & 49.2 & 47.9 & 48.8 & 50.2 & 48.7 \\
\hline \multirow{4}{*}{2014} & $\mathrm{n}$ & 8022 & 7606 & 8267 & 8210 & 8222 & 8108 & 1539 & 6790 & 8082 & 8108 & 8094 & 7200 \\
\hline & mean & 155.04 & 46.27 & 23.98 & 22.69 & 43.59 & 45.42 & 302.75 & 56.63 & 9.01 & 163.4 & 12.82 & 47.51 \\
\hline & SD & 5.4 & 7.17 & 4.69 & 5.68 & 9.21 & 6.71 & 44.93 & 20.3 & 0.8 & 23.55 & 4.1 & 10.67 \\
\hline & $\begin{array}{l}\text { T-score } \\
\text { (National comparison) }\end{array}$ & 50.4 & 49.4 & 50.6 & 49.3 & 48.3 & 49.7 & 47.3 & 49.4 & 48.3 & 48.7 & 49.9 & 48.9 \\
\hline \multirow{4}{*}{2015} & $\mathrm{n}$ & 8042 & 7798 & 8205 & 8156 & 8195 & 8115 & 1373 & 7170 & 8082 & 8130 & 8116 & 7496 \\
\hline & mean & 155.07 & 46.1 & 23.89 & 22.98 & 44.11 & 45.89 & 304.14 & 57.34 & 8.99 & 166.02 & 12.84 & 48.12 \\
\hline & $\mathrm{SD}$ & 5.35 & 6.97 & 4.57 & 5.81 & 9.37 & 6.83 & 45.63 & 20.37 & 0.82 & 23.63 & 4.12 & 10.69 \\
\hline & $\begin{array}{l}\text { T-score } \\
\text { (National comparison) }\end{array}$ & 50.4 & 49.1 & 50.5 & 49.5 & 48.6 & 49.7 & 46.8 & 49.6 & 48.1 & 49.5 & 50 & 49.1 \\
\hline \multirow{4}{*}{2016} & $\mathrm{n}$ & 7977 & 7783 & 8043 & 7947 & 8004 & 7908 & 1446 & 6982 & 7818 & 7906 & 7913 & 7256 \\
\hline & mean & 155.06 & 46.34 & 24.15 & 23.41 & 44.39 & 46.77 & 303.34 & 57.97 & 8.92 & 167.47 & 12.97 & 49.2 \\
\hline & SD & 5.43 & 6.99 & 4.67 & 5.69 & 9.3 & 7.02 & 45.53 & 20.77 & 0.79 & 23.25 & 4.19 & 10.69 \\
\hline & $\begin{array}{l}\text { T-score } \\
\text { (National comparison) }\end{array}$ & 50.4 & 49.5 & 50.9 & 49.9 & 48.9 & 50.2 & 46.6 & 49.6 & 48.9 & 49.7 & 50.3 & 49.7 \\
\hline \multirow{4}{*}{2017} & $\mathrm{n}$ & 7805 & 7565 & 7924 & 7855 & 7896 & 7817 & 1101 & 7104 & 7771 & 7825 & 7801 & 7241 \\
\hline & mean & 155.09 & 46.36 & 24.11 & 23.81 & 44.7 & 46.89 & 303.77 & 57.92 & 8.95 & 165.67 & 13.06 & 49.27 \\
\hline & SD & 5.42 & 7.21 & 4.75 & 5.78 & 9.25 & 6.91 & 49.63 & 19.65 & 0.82 & 23.93 & 4.26 & 10.83 \\
\hline & $\begin{array}{l}\text { T-score } \\
\text { (National comparison) }\end{array}$ & 50.4 & 49.5 & 50.6 & 50.1 & 48.8 & 50.2 & 46.2 & 49.4 & 48.2 & 48.8 & 50.2 & 49.4 \\
\hline \multirow{4}{*}{2018} & $\mathrm{n}$ & 7541 & 7385 & 7633 & 7579 & 7614 & 7553 & 983 & 7021 & 7472 & 7548 & 7535 & 7108 \\
\hline & mean & 155.12 & 46.32 & 24.06 & 23.91 & 44.95 & 47.47 & 304.56 & 59.88 & 8.89 & 168.48 & 12.96 & 50.14 \\
\hline & $\mathrm{SD}$ & 5.36 & 7.11 & 4.81 & 5.78 & 9.34 & 7.26 & 50.88 & 20.31 & 0.8 & 23.63 & 4.26 & 10.78 \\
\hline & $\begin{array}{l}\text { T-score } \\
\text { (National comparison) }\end{array}$ & 50.4 & 49.6 & 50.4 & 50.1 & 48.7 & 50.1 & 45.9 & 50 & 48.5 & 49.3 & 50 & 49.6 \\
\hline \multirow{4}{*}{2019} & $\mathrm{n}$ & 7328 & 7237 & 7404 & 7367 & 7375 & 7318 & 327 & 7084 & 7277 & 7327 & 7312 & 6868 \\
\hline & mean & 155.09 & 46.53 & 24.12 & 24.15 & 45.67 & 47.38 & 307.41 & 58.4 & 8.92 & 169.36 & 12.99 & 50.13 \\
\hline & $\mathrm{SD}$ & 5.43 & 7.12 & 4.7 & 5.86 & 9.46 & 6.72 & 48.14 & 19.34 & 0.8 & 23.12 & 4.3 & 10.91 \\
\hline & $\begin{array}{l}\text { T-score } \\
\text { (National comparison) }\end{array}$ & 50.4 & 49.6 & 50.7 & 50.8 & 49.3 & 50.1 & 45.9 & 50 & 48.6 & 49.8 & 50.1 & 49.9 \\
\hline
\end{tabular}




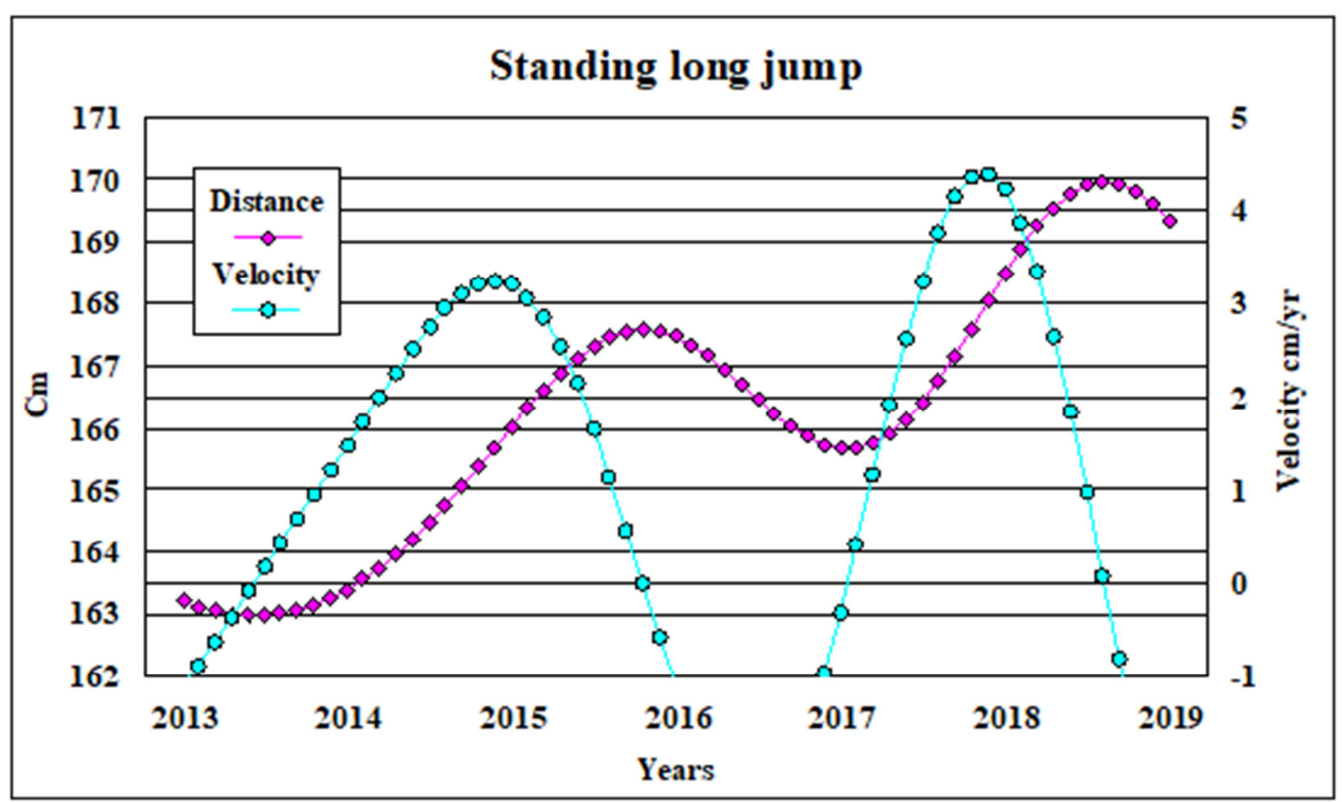

Figure 6. The secular change curve of standing long jump by wavelet interpolation model (The second grade at a junior high school girls).

\subsection{Proposal for a Multi-year Span Evaluation Chart with the $O$ Model of Physical Strength}

The graphs in Figures 7 to 9 are for the repeated side step, sit-up, and 20-m shuttle run in second year junior high school girls, which showed rising trends over the years as mentioned in the Analysis section. In Figures 10 to 12, the wavelet interpolation model was applied to the trends over time in the sit-and-reach, 50-m dash, and standing long jump. The wavelet interpolation model was further applied to the trends in mean values and to the figures in a mean value 5-step model of the standard deviations of those means. These are the described evaluation charts. We named these charts multi-year span evaluation charts. This chart is standardized to the model of City $\mathrm{O}$, and figures showing the national mean values are fit onto that chart in these six graphs. The fact that the national mean values trend along with the mean values in this chart is evidence showing that they are almost the same. Moreover, the trends over the years in physical strength in second year junior high school girls in the $\mathrm{O}$ model show mostly rising trends, and are rising trend evaluation charts. Thus, this shows that national values are similar to the mean values of the $\mathrm{O}$ model chart from around 2015 or 2016 . This could be said to be evidence showing a rising trend over years in physical strength in City $\mathrm{O}$.

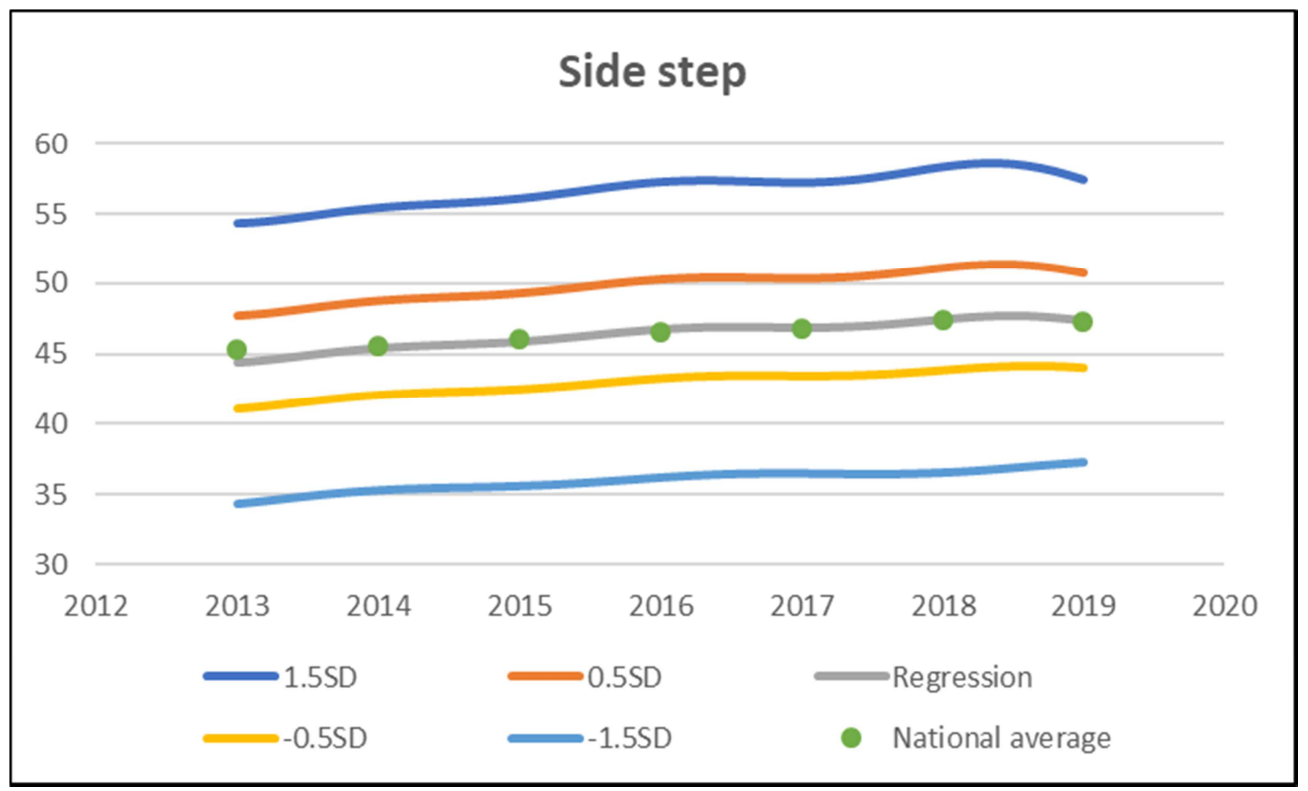

Figure 7. Multi-year span evaluation chart of side step in O model (The second grade at a junior high school girls). 


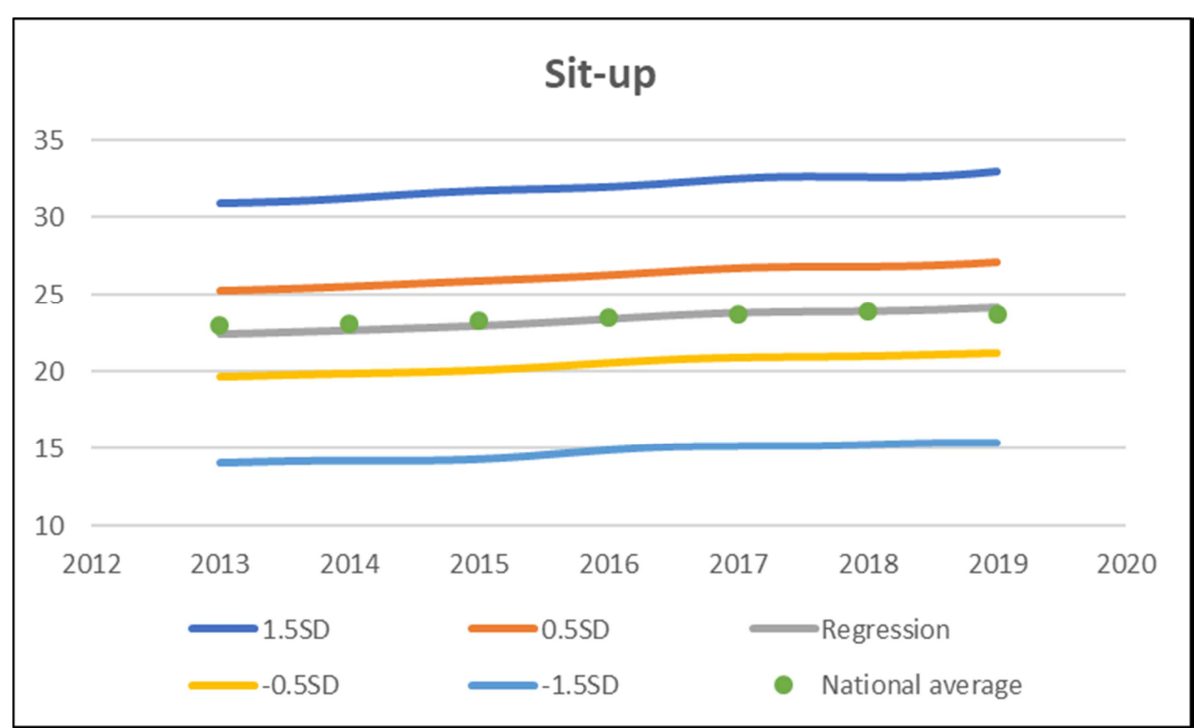

Figure 8. Multi-year span evaluation chart of sit-up in O model (The second grade at a junior high school girls).

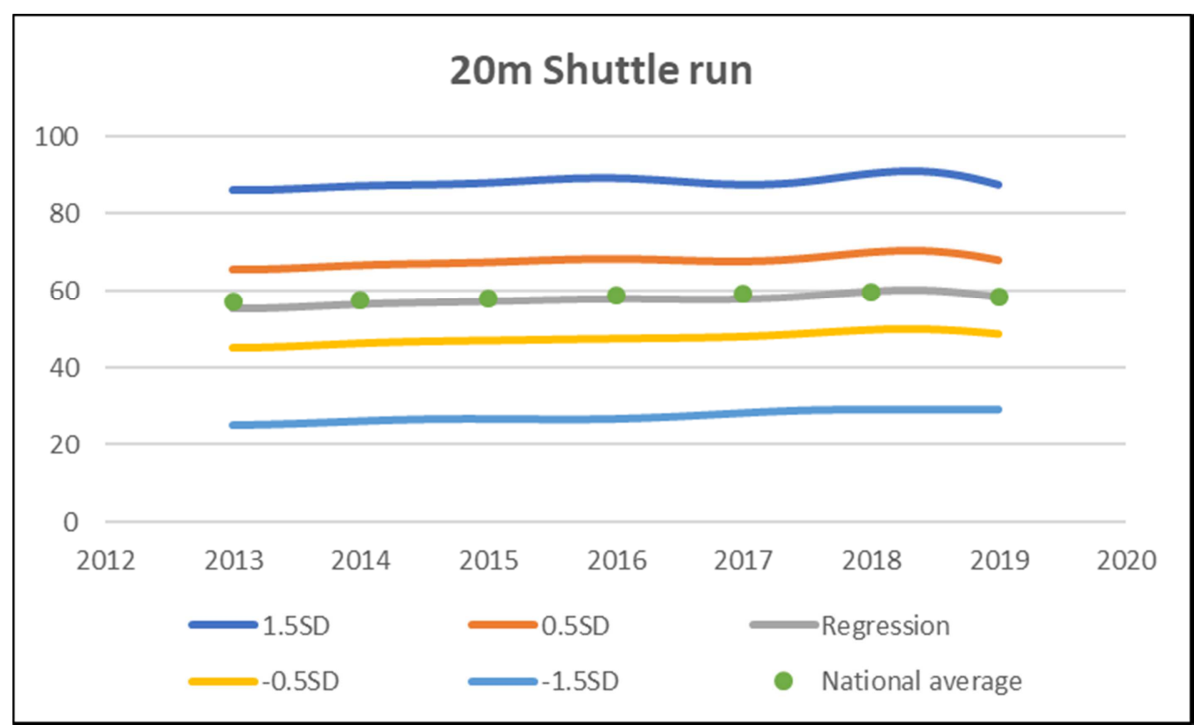

Figure 9. Multi-year span evaluation chart of $20 \mathrm{~m}$ shuttle run in O model (The second grade at a junior high school girls).

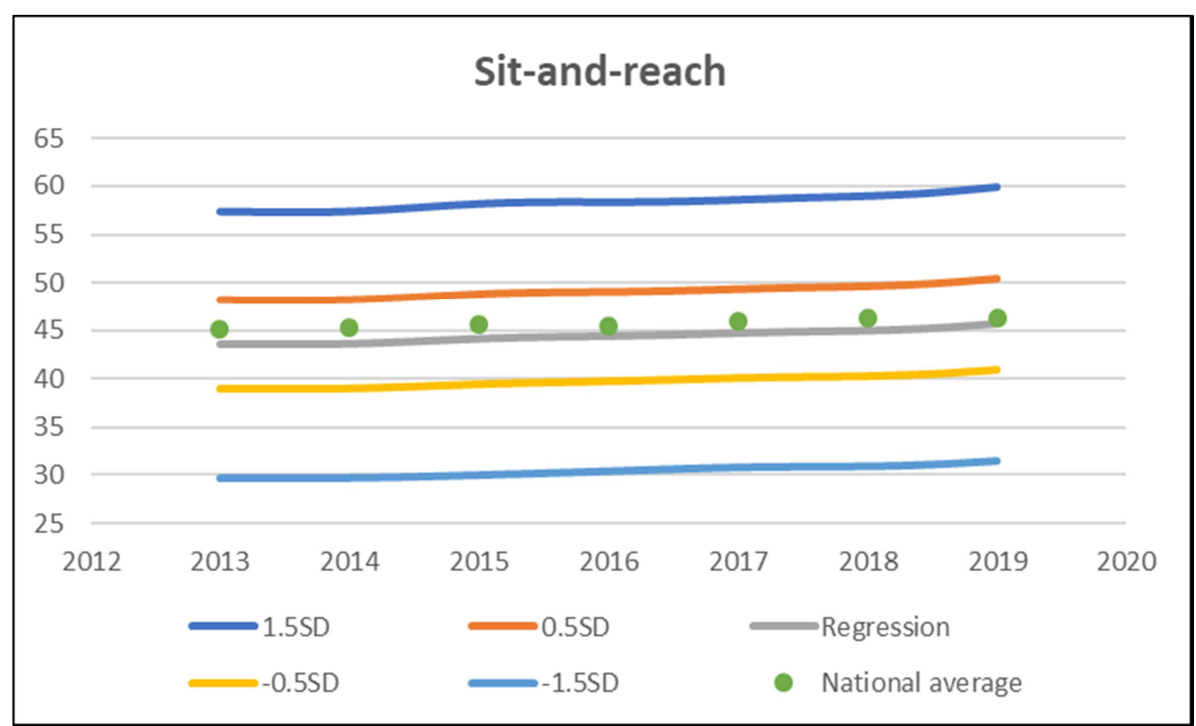

Figure 10. Multi-year span evaluation chart of sit-and-reach in O model (The second grade at a junior high school girls). 


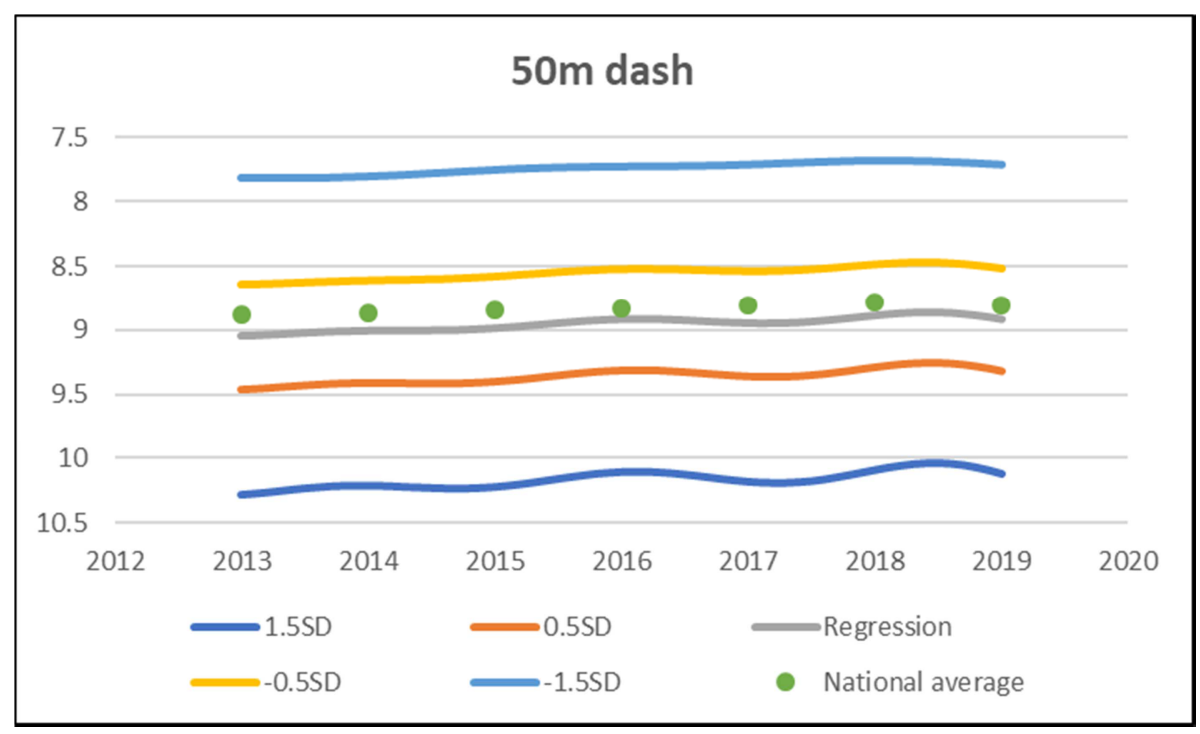

Figure 11. Multi-year span evaluation chart of 50m dash in O model (The second grade at a junior high school girls).

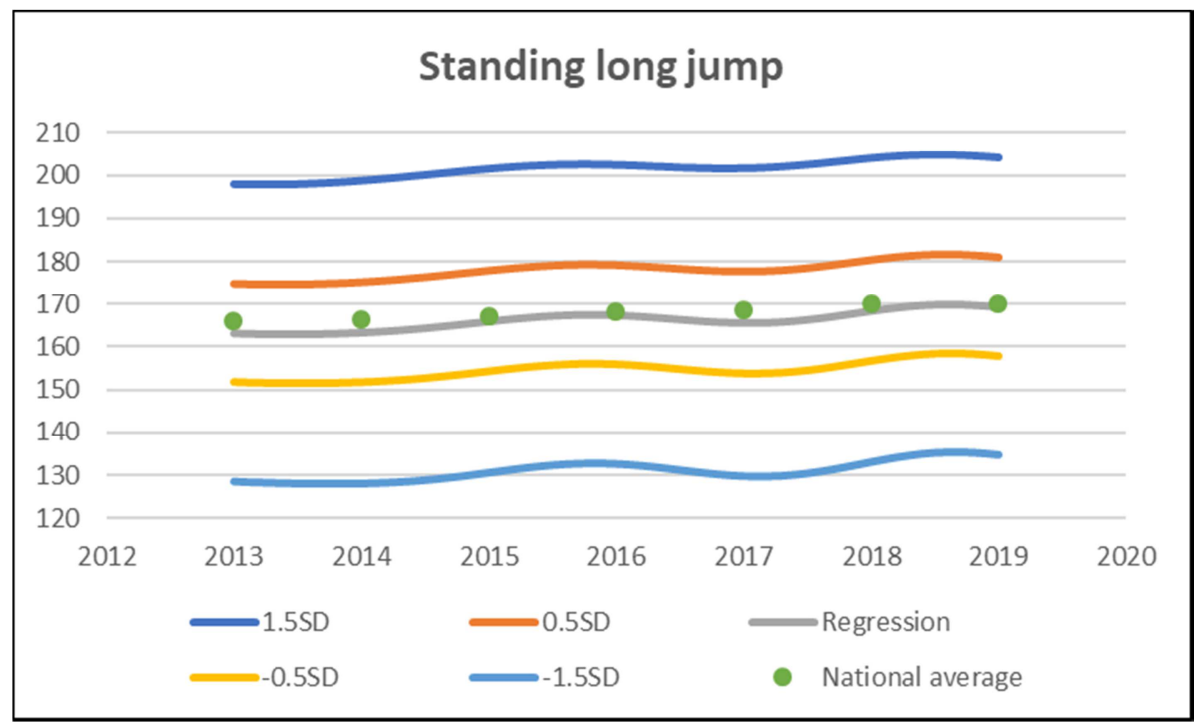

Figure 12. Multi-year span evaluation chart of standing long jump in $O$ model (The second grade at a junior high school girls).

\section{Discussion}

A large number of studies on physique and physical strength have been reported at the university bulletin level. In a classic study [14], findings based on a correlation analysis of physique and physical strength suggested involvement of physique, in particular height, on physical strength. Recently, however, Fujii [15] pointed out that the correlation between height and physical strength is not that high, and that height and motor ability are independent phenomena. Of course, this is meaningful in terms of a survey on physique and physical strength/motor ability, and understanding at the level of physical strength and motor ability seems necessary for the school years today. Given these circumstances, it could be considered important to have an understanding of how physical ability in school-age children has trended in recent years by analyzing the changes over time in physical strength and motor ability. With regard to the $1980-1985$ peak and subsequent decline in physical strength based on trends over years in physical strength and motor ability, Nishijima [4, 5] evaluated a time span from the increase in physical strength and motor ability brought by the Tokyo Olympics until the current declining phase, and marked it as a declining trend in the physical strength of young people. In recent years, it is a fact that the decline in physical strength has had an effect on surveys of physical strength and motor ability during school age, even narrowed down to the fifth grade of elementary school and the second year of junior high school with a view to the background of national tests.

Shimojo et al. [16] investigated the annual changes in physique, muscle strength, and motor ability in university students over 26 years, from the peak of physical strength in 1984 until 2010 and, although there were some missing years, the trends over time in physical strength by body type classification based on BMI. However, statistical stability is not obtained in category analysis using data for a single university, and limitations to the study must be recognized. 
Even in studies of university students, it is difficult to ensure the amount of data to generalize an evaluation chart of physical strength and motor ability. The drawbacks of that analytical method are a major problem. This is the same in studies of physical strength and motor abilities in school-age children, and it is impossible to statistically generalize with data from a single school. Even with surveys undertaken at the prefecture or municipal level like this one, while large amounts of data are guaranteed, the analytical method for those large amounts of data is a problem. A traditional analytical method is a $\mathrm{T}$ score $\{50+10 \times$ (national mean local mean)/national standard deviation $\}$, and while it is a deviation value, the $\mathrm{T}$ score relies too much on acceptance of things that are unknown. This leads to the phenomenon of competition for ranking among all prefectures and municipalities in the nation. These numbers are like magic, and even if the difference in mean values is divided by the standard deviation, the meaning of those numbers is nothing more than a deviation value that suggests mere order.

In particular, how much meaning is there to a deviation value ( $\mathrm{T}$ score) in physique, physical strength, and motor ability? There is some concern about considering it to be equivalent to a deviation value in academic ability. Unquestionably, academic ability as well as physical strength and motor ability are all things that cannot be seen with the eye. The methods used to quantify those abilities are academic tests and physical strength/motor ability tests. For example, determining relative levels of academic ability is an inevitable procedure for the purpose of giving common tests to select who gets into university. However, attaching an order to relative levels in physical strength and motor ability is a different phenomenon than relative levels of academic ability, in that the purpose differs. The purpose of surveys of physical strength and motor performance is not to rank relative levels but to assess whether school-age children have acquired physical abilities corresponding to their living environment and behavior patterns. Naito [17] indicated that understanding current physical strength is important in order to increase physical strength in order to lead a healthy life. Of course, the survey results for physical strength and motor ability in this study are useful as data in discovering sports talent. Obviously, depending on the purpose of use, there are some cases when it would be useful to rank the relative levels of these survey results.

Therefore, since the trends over seven years were surveyed in this study, considering the things mentioned above we explored an evaluation in a longer span rather than constructing evaluations for single years. This evaluation is an evaluation chart that considers trends over years, and becomes a true evaluation of physical strength in school-age children in recent years. In this study the wavelet interpolation model was applied to trends over years, especially to the physical strength of second year junior high school girls. The results differ in dimension from judgments based on viewing conventional polygonal line graphs with the naked eye, and an increasing trend was clearly shown based on scientific judgment. The grounds for that judgment are demonstrated in the behavior of the velocity curve. Specifically, what the increasing trend in physical strength over the years in second year junior high school girls showed is conjectured to be the results of the City $\mathrm{O}$ action plan. Behind the increasing trend over the years in second year junior high school girls but not boys is that while girls originally had lower levels of activity than boys, their activity was stimulated by the action plan. Moreover, those years are the years of late puberty for girls, and the time after the peak in physical development velocity in girls, which is around the second year of junior high school, is a time that is approaching the developmental peak in physical strength. Thus, it may be conjectured that this is tied to increased physical strength. Hence, the multi-year span evaluation chart for physical strength and motor ability is proposed here as the O model.

When constructing an $\mathrm{O}$ model multi-year span evaluation chart, it is important to prepare an evaluation line that distinguishes between evaluation bands. Until recently, the method of analyzing trends over years has been to apply a least squares approximation polynomial devised by Fujii [13], but it had the problem that it did not go through the observation points, and so while it was sufficiently effective for seeing simple trends, in this study we decided to apply the wavelet interpolation model, which can pass through the observation points, rather than the least squares approximation since this study had a relatively short span of seven years. The reason for applying the wavelet interpolation model is that with first-degree functions only linearly increasing compositions can be verified, but with second degree or higher functions it is thought that the composition of the changes in increases can be captured in detail. The wavelet interpolation model does not have the characteristics of a formula applied as mathematical functions, and so is effective in that the trends for changes in physical strength over years do not depend on functions. In other words, the trends in physical strength changes over years can be grasped as a model of true changes. This efficacy of the wavelet interpolation model was previously explained by Fujii [18].

In this study, the wavelet interpolation model was applied to the upward trends over a number of years in the physical strength of second year middle school students. Wavelet interpolation was applied to the reference values of a 5-step evaluation from the means and standard deviations for physical strength in the respective years, and multi-year span evaluation charts were constructed. It was possible to make comparisons over time with the national level by applying national values published by the Ministry of Education, Culture, Sports, Science and Technology to the multi-year span evaluation chart based on the Kansai area as the O model. This method was used for the first time in this study, and by conversely evaluating the national figures it was seen that the current physical strength in school-age children in City $\mathrm{O}$ had no major differences from the national level. Furthermore, an effect from the City $\mathrm{O}$ action plan was indicated in the rising trend over time in second year junior high school students, demonstrating the effectiveness of the multi-year span evaluation chart. 


\section{Conclusion}

In examining the effects of the action plan implemented by City $\mathrm{O}$ in the Kansai area on the decrease in physical strength, this study analyzed the changes over time in school-age children from a continuous seven-year survey of physique, physical strength, and motor ability in fifth grade students and second year junior high school students. We examined whether the trends were rising or falling by applying the wavelet interpolation model to the trends over seven years. The results showed a rising trend over the years in the physical strength of second year junior high school girls. Behind the rising trend over time is conjectured to be the effect of the action plan of City O. However, the background for the improving trend seen over the years in second year junior high school girls but not boys includes that girls were originally less active than boys and but were encouraged to be active by the action plan. Moreover, those years are the years of late puberty for girls. The time after the peak in physical development velocity in girls, which is around the second year of junior high school, is a time that is approaching the developmental peak in physical strength. Thus, it may be conjectured that factors in the action plan influenced the increase in physical strength. In this study, a new evaluation chart that considers trends over years was established using the wavelet interpolation model, and the efficacy of the multi-year span evaluation chart for physical strength was proposed as the $\mathrm{O}$ model.

\section{References}

[1] H. Nakai, S. Demura, "Influence of habitual exercise on physique and physical fitness in adolescent male students: From an examination of three-year longitudinal data," Japanese Society of Physical Education, Vol. 39 (4), pp. 287-303, 1994.

[2] S. Shimada, S. Demura, Y. Ikemoto, S. Yamaji, M. Minami, Y. Nagasawa, "Cross Sectional Study of The Relationship between Physical Fitness and Life Style," Health-Status in Male Students at a National College of Technology, Japan Society of Physiological Anthropology, Vol. 8 (3), pp. 109-117, 2003.

[3] S. Shimada, S. Demura, Y. Nagasawa, M. Minami, J. Matsuzawa, "Influence of The Difference of Continuous Exercise Enforcement Frequencies on Physique and Physical Fitness of Male Students at a National College of Technology: With an Examination of Three-Year Longitudinal Date," Japan Society of Physiological Anthropology, Vol. 11 (2), pp. 69-74, 2006.

[4] H. Hatta, "Annual trends in physical strength of university students-The University of Tokyo," Journal of Health, Physical Education and Recreation, Vol. 52, pp. 39-42, 2002.
[5] T. Nishijima, "Declining trend in the physical strength of adolescents," Journal of Health, Physical Education and Recreation, Vol. 52, pp. 4-14, 2002.

[6] T. Nishijima, "Current status of physical strength in children," Child, Growth and Development, Vol. 1, pp. 13-22, 2003.

[7] R. Sasaki, "Annual trends in physical strength of university students-Keio University," Journal of Health, Physical Education and Recreation, Vol. 52, pp. 43-47, 2002.

[8] T. Matsumoto, "Annual trends in physical strength of university students-Tsukuba University," Journal of Health, Physical Education and Recreation, Vol. 52, pp. 48-51, 2002.

[9] K. Fujii, Kim J-D, K. Kasuya, "Verification regarding Optimum Physical Fitness for Balance of Body Composition During Junior High School Period - Analysis based on Korean junior high school students in boys -," The Korea Journal of Sports science Vol. 24 (6), pp. 1409-1418, 2015.

[10] K. Hayakawa, K. Fujii, K. Kasuya, T. Kondo, “An Approach to Disease Guidelines based on Standardization of Body Composition Balance," International Society for Standardization Studies, Vol. 14 (1), pp. 1-17, 2016.

[11] K. Fujii, Y. Matuura, "Analysis of the Velocity Curve for Height by the Wavelet Interpolation Method in Children Classified by Maturity Rate," American Journal of Human Biology, Vol. 11, pp. 13-30, 1999.

[12] K. Fujii, "Confirmation regarding secular trend of physical growth in Korean school students by wavelet interpolation method: analysis from secular trend of age at MPV of height and weight," The Journal of Education and Health Science, Vol. 54 (2), pp. 129-140, 2008.

[13] K. Fujii, "Verification Regarding Secular Trend of Height Growth and The Maximum Peak Velocity during Adolescence," International Journal of Sport and Health Science, Vol. 7, pp. 103-112, 2009.

[14] T. Mizuno, "Japanese Physical Fitness Standard Table - By Height-Based Regression Evaluation Method," University of Tokyo Press, 1980.

[15] K. Fujii, "Exploration of Human Resource Elements of Sports Athletes - Sports Talent and Tall Height - ," Production Management, Vol. 27 (1), pp. 161-169, 2020.

[16] H. Shimojo, Y. Nakata, M. Tomikawa, H. Takagi, H. Soya, "Trends in body mass index and physical fitness of Japanese university students over 26 years and the association between these parameters," Japan J. Phys. Educ. Hlth. Sport Sci, Vol. 58, pp. 181-194, 2013.

[17] H. Naito, "Current state and issues in physical strength in children." National Strength and Conditioning Association Japan, Vol. 18 (1), pp. 2-6, 2011.

[18] K. Fujii, "A scientific approach to growth and development-Physical information science for growth and development \& health-," Sankeisha, 2006. 